\title{
Study of Adiponectin Promotor Methylation status in patients with Non Alcoholic Fatty Liver Disease \\ M.A.Abd El-moaty ${ }^{1}$, I.A.Elsayed ${ }^{1}$, Y.Saad ${ }^{2}$, S.K.Eliwa ${ }^{1}$ and N.E.Abd El-Maksoud ${ }^{1}$ \\ ${ }^{1}$ Medical Biochemistry,Molecular Biology,Dept.,Faculty of Medicine, Benha Univ., Benha, Egypt \\ ${ }^{2}$ Endemic Medicine, hepatogastroenterology, Dept., Faculty of Medicine, cairoUniv., cairo, Egypt \\ E-mail:dr.namaa_elsayed@yahoo.com
}

\section{Abstract}

Nonalcoholic fatty liver disease (NAFLD) is one of the most common causes of liver disease globally, and it is likely to overtake alcoholic liver disease as the primary cause of end-stage liver disease in the coming decades. It affects both adults and children. In light of the significant hurdles in the prevention and control of NAFLD, recent research have shown that detecting adiponectin promotor methylation may be useful in NAFLD diagnosis, prognosis, and treatment.. Adiponectin (an adipose tissue hormone) induces apoptosis in hepatocytes, which has an anti-steatotic effect. Several samples from patients with NAFLD have been shown to exhibit adiponectin promotor methylation (NAFLD) so it can be used as non- invasive tool for NAFLD diagnosis.

\section{Keywords: Non Alcoholic Fatty Liver Disease, Adiponectin, Methylation.}

\section{Introduction}

After all other liver etiologies have been ruled out, non-alcoholic fatty liver disease (NAFLD) is caused by fat accumulation in the liver [1]. Hepatic fat accounts for more than $5-10 \%$ of entire liver weight in NAFLD. The majority of patients with NAFLD have merely an increase in liver fat (simple steatosis). Nonalcoholic steatohepatitis (NASH) is a disorder in which some individuals develop hepatic inflammation, and up to $20 \%$ of patients acquire progressive hepatic fibrosis, which can lead to liver cirrhosis or failure, as well as hepatocellular cancer [2].

NAFLD is a silent disease that affects the Egyptian people. It affected $20 \%-30 \%$ of the general population worldwide, but it can affect up to $50 \%$ of the population in obese people or those with diabetes mellitus. According to previous studies, steatosis is found in $70 \%$ of obese patients and $35 \%$ of non-obese patients, while NASH is found in 18.5 percent of obese patients and $3 \%$ of non-obese patients. Fatty liver disease might affect up to 75 percent of patients with type 2 diabetes mellitus (DM2) [3].

The total incidence of NAFLD in adolescents has reached about $10 \%$, with $17 \%$ of teenagers and $40 \%-$ $70 \%$ of obese youngsters suffering from the disease [4].

NAFLD pathogenesis has been linked to a number of risk factors, including advanced age, obesity, insulin resistance, and hyperlipidemia, as well as the involvement of pro- and anti-inflammatory cytokines [5].

A number of recent results have highlighted the importance of adipose tissue as an active endocrine organ that produces adipokines such as adiponectin (APN), leptin, resistin, and visfatin, all of which are involved in the progression and pathogenesis of NAFLD [6].

Hepatocytes respond to adiponectin by increasing free fatty acid oxidation while decreasing gluconeogenesis, FFA inflow, and de novo lipogenesis. Hepatocytes are protected against apoptosis by it. It has anti-inflammatory and anti-fibrotic properties by acting on Hepatic stellate cells (HSC), Kupffer cells, and perhaps sinusoidal cells. It works to reduce inflammation by inhibiting pro-inflammatory cytokines (TNF- $\alpha$ and IL-6) and activating anti-inflammatory cytokines (IL-10) [7].

Adiponectin's anti-fibrotic activity is primarily done by inhibiting HSC activation and proliferation while inducing apoptosis. TGF-, connective tissue growth factor, and collagen are all downregulated by adiponectin, which favours matrix breakdown [8].

A liver biopsy is the gold standard for diagnosing $\mathrm{NASH}$. This treatment, on the other hand, is invasive, pricey, and linked to uncommon but potentially dangerous consequences and sampling errors; thus, it is ineffective as a screening tool [2]. As a result, detecting adiponectin promoter methylation could be useful in NAFLD diagnosis, prognosis, and treatment.

\section{Subject and methods}

This study was carried out between July 2018 and July 2021 after approval of the study scheme by the research ethical committee of Benha Faculty of Medicine and obtaining informed consent from the included subjects. The study included 49 subjects of both sex selected from Endemic Medicine Department, Faculty of Medicine, Cairo University Hospital.

The subjects were categorized into 2 groups: patient group: included 34 patients diagnosed as nonalcoholic fatty liver diseae patients by clinical, radiological and histopathological examinations examinations and control group: included 15 persons, age and sex matched, with normal liver.

All patients were subjected to full history taking with attention to: special habits including, obesity and sedentary life and many investigations, diagnostic Liver biopsy for histopathology and Methylation specific polymerase chain reaction (MSP) for detection of adiponectin promotor methylation status.

Blood samples were obtained from all individuals. Blood samples were collected into EDTA vacutainers. From each blood sample $(1 \mathrm{ml})$ was transferred immediately into Eppendorf tubes to be stored at - 
$80^{\circ} \mathrm{C}$, for later processing. Extraction of DNA from Peripheral blood samples: using QIAmp DNA blood mini kit (QIAGEN, Germany) according to manufacturer's instructions. Bisulfite treatment of extracted DNA was done by using The EZ DNA Methylation-Gold Kit (ZYMO RESEARCH, USA), according to manufacturer's instructions. Methylation specific PCR by using HotStarTaq Master Mix Kit (QIAGEN, Germany) and Specific primer sets for either methylated or non-methylated products of adiponectin gene promoter. The PCR product was separated by gel electrophoresis, stained with ethidium bromide and visualized by UV irradiation, for detection of specific bands.

\subsection{Statistical analysis}

The collected data were summarized in terms of mean \pm Standard Deviation (SD) and range for quantitative data; and frequency and percentage for qualitative data. Comparisons between cases and control were carried out using the student $\mathrm{T}$ - test, to compare quantitative data between two groups and Chi- squared $\left(\chi^{2}\right)$ test, to compare proportions of two or more groups. Pearson correlation was used to estimate the correlation between ADPN-M, ADPN-U and age of the studied group. The corresponding test statistics were calculated and the corresponding P-values were obtained. P-value 0.05 was considered statistically significant, while P-value > 0.05 was considered statistically non-significant. Analysis is performed using the Statistics Program for Social Sciences (SPSS) and Microsoft Office Excel is used for the data processing and data analysis.

\section{Results}

49 persons were included in the study. 34 patients were diagnosed with NAFLD while the remaining 15 , having no evidence of NAFLD, were included as controls.

Demographic data of studied groups

The baseline characteristics of the study population are presented in Table-1. There was insignificant difference between cases and control groups regarding age $(\mathrm{p}=0.937)$, there is significant difference between cases and control groups regarding gender $(\mathrm{p}=0.022)$.

Table (1) Demographic data of the studied groups.

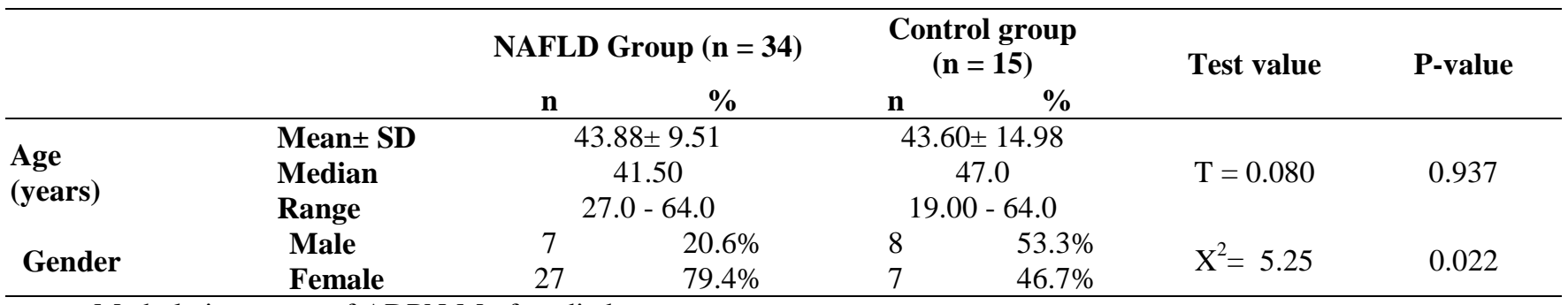

Methylation status of ADPN-M of studied groups

ADPN-M, was studied and Methylation status was analyzed in blood samples. The Methylation status was significantly higher $(\mathrm{p}<0.003)$ in NAFLD patients as compared to controls table $(2)$.

Table (2) Comparison between the studied groups regarding degree of methylation.

\begin{tabular}{|c|c|c|c|c|c|c|}
\hline & \multicolumn{2}{|c|}{$\begin{array}{l}\text { NAFLD Group } \\
(\mathbf{n}=\mathbf{3 4 )}\end{array}$} & \multicolumn{2}{|c|}{$\begin{array}{c}\text { Control group } \\
(\mathbf{n}=\mathbf{1 5})\end{array}$} & \multirow[t]{2}{*}{ Test value } & \multirow[t]{2}{*}{ P-value } \\
\hline & Mean & \pm SD & Mean & $\pm \mathrm{SD}$ & & \\
\hline METH \% $(\Delta \Delta C T)$ & 53.68 & 18.82 & 40.03 & 10.98 & 3.18 & 0.003 \\
\hline
\end{tabular}

SD: Standard deviation, P $0.05 \leq$ significant, P > 0.05 non-significant, analysis done by independent samples Student T test.

\section{Discussion}

NAFLD is a global health issue that affects over $25 \%$ of the world's population. The Middle East has the greatest prevalence rate of NAFLD $(31.79 \%)$, while Africa has the lowest incidence rate (13.48 percent) (9).

In Egypt, a cross-sectional study showed that the prevalence of NAFLD in children and adolescents was $15.8 \%$, and the prevalence of NAFLD increased with age, from less than $20 \%$ under 20 to over 60 for more than $40 \%$ of the year, NAFLD is more common in men (31\%) than in women (16\%) [9].

Although liver biopsy is the gold standard for diagnosing NAFLD, it is an intrusive operation with possible risks and complications. As a result, noninvasive diagnostic methods as alternatives to liver biopsy have been proposed [10].

The early identification of high-risk patients by the measurement of a number of specific biomarkers is critical to a successful preventative programme. As treatment options for NASH patients at risk of progression are applied, biomarkers are becoming increasingly important for screening and identifying therapy responses [11].

The subject of genetic variables in NAFLD research is continuously expanding. NAFLD and its severity have been linked in many studies to serum 
adipokines, a group of bioactive proteins released by adipose tissue that have anti-inflammatory and insulinresistance properties. Adiponectin has been shown to protect against NAFLD in several trials [10].

Adiponectin is an adipose tissue-derived hormone that regulates glucose and lipid metabolism to influence whole-body energy homeostasis. Adiponectin improves insulin sensitivity in metabolic tissues by boosting glucose consumption and fatty acid oxidation. Obesity and obesity-related metabolic disorders such as insulin resistance, T2D, and cardiovascular disease are inversely linked with adiponectin blood levels [12].

In our study, there is adiponectin promotor hypermethylation status in patient with NAFLD, this is in line with Previous research which shown that DNA hypermethylation of the adiponectin promoter inhibits adiponectin expression and, as a result, decreases its activity and exacerbates metabolic disorders in obese people [13]. Hypoadiponectinemia was found in women with GDM, according to another investigation. In maternal fat and blood cells, significant changes in locus-specific DNA methylation were detected. The methylation of DNA in GDM offspring was changed [14].

It could be concluded that the hypermethylation starus of aiponectin promotor in NAFLD patients compared to the controls suggests their role as diagnostic non- invasive markers. The results of this study revealed that ADPN-M in blood sample is a potentially useful blood biomarker for early diagnosis of NAFLD.

\section{Conclusion}

It could be cocluded that hypermethylation status of adiponectin promotor in NAFLD patients compared to controls suggests their rolle as diagnostic noninvasive markers, the result of this study revealed that ADPN-M in blood sample is a potentially useful blood biomarker for early diagnosis of NAFLD.

\section{References}

[1] BJ.Perumpail, MA. Khan, ER. Yoo , Clinical epidemiology and disease burden of nonalcoholic fatty liver disease. World J Gastroenterol.vol.23(47),pp.8263 13748,2017.

[2] IH. Borai, Y .Shaker, MM. Kamal ,Evaluation of Biomarkers in Egyptian Patients with Different Grades of Nonalcoholic Fatty Liver Disease. J Clin Transl Hepatol.vol. 5(2),pp.109-118,2017.

[3] AO. Binobaid, MA. Alalwan, AHA .Almalki , Prevalence and Risk Factors of Fatty Liver among Adults. The Egyptian Journal of Hospital Medicine .vol.70 (9),pp. 1552-1567,2018.

[4] A. Deeb, A. Attia, S. Mahmoud, Dyslipidemia and Fatty Liver Disease in Overweight and Obese Children. Journal of Obesity.vol.8,pp. 1570$1666,2018$.

[5] AA. Mohamed, KKE. Gohary, GME. Mashad , Interleukin 10, thyroid status and ferritin are noninvasive prognostic biomarkers for diagnosis of fatty liver disease in children. Journal of International Research in Medical and Pharmaceutical Sciences.vol.8,pp.85-93,2018.

[6] X. Xie, Li H. Yan D, Enhancement of Adiponectin Ameliorates Nonalcoholic Fatty Liver Disease via Inhibition of FoxO1 in Type I Diabetic Rats. Journal of Diabetes Research.vol.0, pp.1700-1750, 2018.

[7] SA. Polyzos, J. Kountouras , CS. Mantzoros , Adipokines in nonalcoholic fatty liver disease, Metabolism Clinical and Experimental.vol. 65,pp.1062-1079,2016.

[8] NK. Saxena , FA. Anania , Adipocytokines and hepatic fibrosis.Trends Endocrinol Metab.vol. 26,pp.153-61,2015.

[9] AM. Hassan, MHA. Elhaw, AA. Ahmed, Value of screening for nonalcoholic fatty liver disease in hyperuricemic patients with normal body mass index by two-dimensional ultrasound: Upper Egypt experience. ALAZHAR ASSIUT MEDICAL JOURNAL.vol.18 ,pp.104-109,2020

[10] EM. Hasan, RA. Abd Al Aziz, D.Sabry, The association of adiponectin gene polymorphisms with susceptibility and progression of NAFLD in a cohort of Egyptian patients. Egyptian Liver Journal .vol.11,pp. 37,2021.

[11] VA. Piazzolla, A.Mangia, Noninvasive Diagnosis of NAFLD and NASH.Vol. 9(4),pp.15,2020.

[12] S. Dias, S.Adam, Y. Abrahams, Adiponectin DNA methylation in South African women with gestational diabetes mellitus: Effects of HIV infection.vol.10,pp.15,1371.

[13] AY.Kim, YJ.Park, X.Pan, Obesity-induced DNA hypermethylation of the adiponectin gene mediates insulin resistance. Nat Commun. vol.6,pp.75-85,2015.

[14] R.Ott, JH.Stupin, K.Melchior, Alterations of adiponectin gene expression and DNA methylation in adipose tissues and blood cells are associated with gestational diabetes and neonatal outcome. Clin Epigenetics .vol.10,pp. 131 ,2018. 